\title{
Die Bedeutung der Faszien im Hochleistungssport
}

\author{
Raimond Igel
}

Im Hochleistungssport werden extreme Anforderungen an den Körper des Athleten gestellt. Die Faszien spielen dabei eine bedeutende Rolle, da sie u. a. einen wesentlichen Teil der Kraftübertragung zwischen Muskel und Gelenk übernehmen sowie Stoßdämpfer- und Energiespeicherfunktion haben. Sowohl im Training als auch im Wettkampf wirken extreme Kräfte, die somit auch das Fasziensystem des Körpers unter extreme Belastungen stellen. Es macht daher Sinn, das fasziale Netzwerk zu trainieren. Geschmeidige, elastische Faszien sind eine nachhaltige Basis für Belastbarkeit im Sinne der Verletzungsprophylaxe und für das Abrufen von körperlichen Höchstleistungen. Die Faszientherapie nach dem Fasziendistorsionsmodell (FDM) kann dabei höchst effektiv zur schnellstmöglichen Wiederherstellung der Leistungsfähigkeit angewandt werden.

\section{Was sind Faszien?}

Faszien sind eine spezielle Form von Bindegewebe, das sich durch den gesamten Körper zieht. Sie bestehen zu einem Großteil aus einem dichten Kollagenfasernetz, Fibroblasten und anderen Zellen. Die Grundsubstanz besteht aus einer wässrigen Lösung von Glykosaminoglykanen, einer aus Eiweiß und Zucker bestehenden Verbindung, die eine gelartige, schleimige Konsistenz verleiht.

Die Faszien erstrecken sich nicht nur von kranial nach kaudal, sondern auch von der obersten Gewebeschicht bis ins Tiefengewebe. So bilden sie ununterbrochen ein 3-dimensionales Gitternetz durch den gesamten Körper bis in die tiefsten Körperstrukturen. Sie sind so auf allen Ebenen des Körpers präsent: Es gibt keine Unterbrechungen in der faszialen Kontinuität.

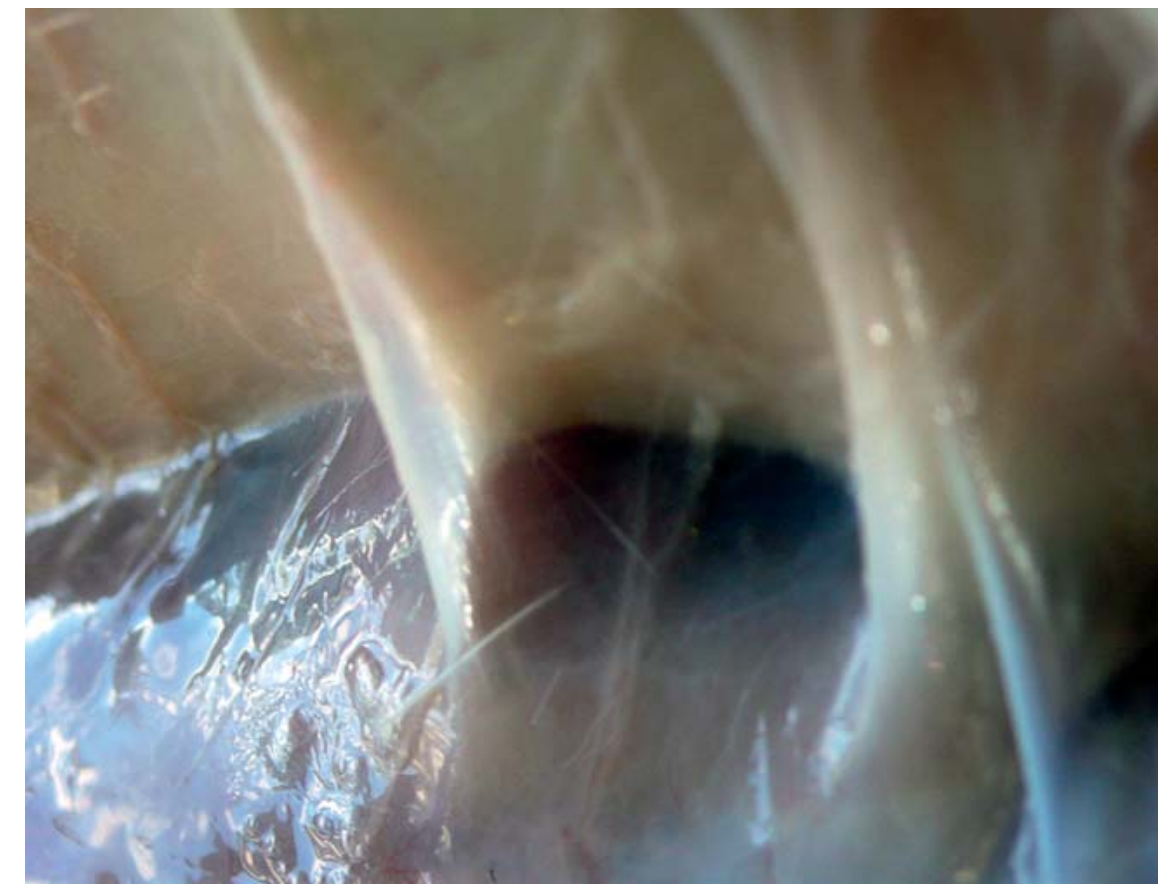

- Abb. 1 Zähe, silberweiß schimmernde, fest am Muskelfleisch haftende Sehne. Bereits die Konsistenz und extreme Anhaftung lassen erahnen, welche Funktionalität, Widerstandsfähigkeit und gleichzeitig auch Anpassungsfähigkeit hinter diesem „faszinierenden“ Gebilde stecken. Quelle: @ fascialnet.com

Neben den meningealen und viszeralen Faszien werden oberflächliche und tiefe Faszienschichten unterschieden. Die oberflächliche Schicht liegt unmittelbar unter der Haut und steht in Verbindung mit dem Unterhautfettgewebe sowie mit Arterien, Venen, Lymphgefäßen und Nerven. Die tiefe Faszienschicht stellt sich komplexer dar. Sie umhüllt die Muskulatur, hält diese damit zusammen und fixiert sie ( $\triangleright$ Abb. 1). Muskelgruppen, sogar einzelne Muskelfasern, werden von der Faszienschicht umgeben und sorgen auf diese Weise für eine optimale Beweglichkeit zwischen den verschiedenen Muskeln. Faszien verlaufen ebenso durch verschiedene Muskelschichten. Des Weiteren umhüllt die tiefe Faszienschicht auch Nerven, Blut-, Lymphgefäße, Knochen und Organe. Auch hier sorgen die Faszien für eine Fixierung bzw. eine optimale Mobilität der umgebenden mit eine lückenlose Verbindung zwischen den einzelnen Körperabschnitten dar. Dieses „Geflecht“ sorgt so für eine gute Stabilität des gesamten Muskel- und Organsystems.

\section{Bedeutung der Faszien im Hochleistungssport}

Faszien existieren (in Form eines speziellen Bindegewebes) im gesamten Körper und haben damit eine fundamentale Rolle für die Aufrechterhaltung aller Körperfunktionen. Sie erfüllen vielfältige Aufgaben, wodurch sie insbesondere bei extremer körperlicher Belastung wie im Hochleistungssport wichtige Schlüsselfunktionen einnehmen. Bislang wurde im Wesentlichen davon ausgegangen, dass
Strukturen untereinander. Sie stellen so- 
allein die Muskulatur für die Leistungserbringung verantwortlich ist. Wenn man allerdings bedenkt, dass Muskulatur allein ohne Faszien einem „Wackelpudding“ gleichkäme, wird der besondere Stellenwert der Faszien mit ihrer Schutz- und Trägerfunktion deutlich erkennbar. Ohne fasziale Unterstützung wäre es gar nicht möglich, Gewichte zu stemmen, den Speer zu werfen, die Kugel zu stoßen, zu springen oder zu laufen.

Eine häufige Annahme war bzw. ist, dass Gelenke vor Überlastungsschäden ausschließlich durch Kräftigung und Stabilitätstraining der Muskulatur geschützt werden können. Auch bei bestimmten Verletzungen, z.B. Zerrungen, die bestimmte Schmerzmuster hervorrufen, wird häufig lediglich ein muskulärer Hintergrund unterstellt. Nahezu provokant wirkt deshalb die Feststellung, dass viele Belastungsschäden, z. B. im Hochleistungssport, eben nicht nur im Muskelgewebe liegen, sondern auch im faszialen Bindegewebe (Fasziennetz) zu suchen sind. Die Muskelfasern sind meist gar nicht betroffen, sondern vielmehr Bänder, Kapseln und Sehnen. Sie sind der extremen sportlichen Belastung - und somit ggf. sogar einer Überbelastung - ausgesetzt, was dann zu diversen Verletzungsmustern führt.

Ein wichtiger Punkt ist ebenso, dass intakte Faszien wesentlich dazu beitragen, dass das Muskelsystem überhaupt positiv wirken kann. Die Faszien gewährleisten, dass die Gelenke stabil bleiben und funktionieren. Das Muskelsystem ist damit eher der Motor der Gelenkbewegung, wird jedoch durch die fasziale Mechanik koordiniert.

Auch erfolgt die Kraftübertragung keineswegs ausschließlich über die Muskulatur. Die Faszien übernehmen hier eine wichtige Weiterleitungsfunktion. Neueste Erkenntnisse zeigen, dass bspw. ca. ein Drittel der Kraft des M. gluteus maximus über die fasziale Verbindung bis ins Knie gelangt. Dies gibt sogar Anlass, das Muskel-Hebel-Gesetz und die Anatomie bzw. Physiologie der Muskelfunktionen zu überdenken.

Eine weitere Schlüsselfunktion ist die Stoßdämpferwirkung der Faszien, die vergleichbar mit einem Hydrauliksystem - entstandene Kräfte auffangen und weiterleiten. So wirken z. B. beim Kugelstoßen Kräfte, die das Doppelte des eigenen Kör- pergewichts betragen und innerhalb kürzester Zeit $(2,5 \mathrm{~m} / \mathrm{s})$ abgefangen werden müssen. Am Beispiel des amtierenden Europameisters David Storl, der alleine im Training ca. 5500 Stöße pro Jahr absolviert, wirken bei einem Körpereigengewicht von $120 \mathrm{~kg}$ bei der Stoß-/Stemmbewegung im Ring ca. $240 \mathrm{~kg}$ auf die Faszienstruktur des linken Kniegelenks. Dabei ist die Pufferfunktion durch die gelartige Faszienstruktur zum Schutz der Gelenke von großer Bedeutung. Bei einer Überbelastung, die durch die einwirkenden Schwerkräfte erfolgt, kann es zu einer Schädigung der Faszienstruktur (Distorsion) kommen. David Storl unterzog sich einer faszialen Behandlung (Kontinuumdistorsion). Dank der geräteunterstützten Faszientherapie mit dem „Fazer“ der Firma Artzt hat er die EM in Zürich 2014 erfolgreich bestritten.

Dass das Fasziennetz eines der reichhaltigsten Sinnesorgane darstellt, ist ebenso eine wichtige Erkenntnis für die trainingsstrategischen Überlegungen im Hochleistungssport. Durch die zahlreichen Propriorezeptoren, die das Fasziennetz besiedeln, sind sie bedeutend für die Wahrnehmung des eigenen Körpers, der Haltung und Bewegung. Dies ist elementar wichtig für die Behandlung von Athleten mit unterschiedlichen Verletzungsmustern. Davon abgeleitet lässt sich vermuten, dass Verletzungen wie Zerrungen, Faserrisse, Traumen, Operationen und Adhäsionen immense Auswirkungen auf die Propriorezeptoren der Faszienschicht (Kapsel-Band-Apparat) haben, was wiederum Störungen in der gesamten Bewegungskoordination und den Bewegungsketten nach sich zieht. Zur Verdeutlichung: Bei einem Athleten, der sich beim Dauerlauf eine Bänderüberdehnung (Distorsion) zugezogen hat und nach diversen klassischen Behandlungen (Physiotherapie, Salben-, Tapeverbände) noch immer das Gefühl einer Instabilität im Sprunggelenk hat, liegt nach der beschriebenen Erkenntnis der Verdacht nahe, dass die Propriorezeptoren in ihrer eigentlichen Aufgabe noch immer gestört sind.

Bereits der Begründer der Osteopathie, Andrew Taylor Still, schrieb den Faszien eine bedeutende Rolle zu: „Ich kenne keinen Teil des Körpers, der es den Faszien als Forschungsfeld gleich tun kann. Ich glaube, dass sich beim Studium der Faszien mehr reichhaltige und goldene Einsichten 
auftun werden. Durch die Funktion der Faszien leben wir und durch ihr Versagen sterben wir.“ [6]

\section{Therapeutisches Konzept}

Den bisherigen Erkenntnissen der Faszienforschung und -therapie folgend ist es unerlässlich, das Fasziendistorsionsmodell (FDM) bei der Befunderhebung und Behandlung einzubeziehen. Mit dem primären Effekt einer erfolgreichen FDM-Behandlung - der vollen Wiederherstellung der Leistungsfähigkeit - ist ein weiterer Effekt verbunden: Die Störung inkl. der dazugehörigen Schmerzmuster ist in der Regel innerhalb kürzester Zeit vollständig therapiert und der Athlet damit schnellstmöglich wieder voll einsatzfähig - im Hochleistungssport meist ein entscheidender Aspekt. Die beschriebenen Aspekte sind wesentlich im Rahmen der Forschungsarbeit von einem der führenden Faszienforscher, Dr. Robert Schleip, entstanden bzw. nachgewiesen worden. Die Forschungsergebnisse regen stark dazu an, die Therapie von Sportverletzungen zu überdenken.

Neben der Faszientherapie zur Behandlung von auftretenden Störungen ist ein weiteres wichtiges Gebiet das Faszientraining. Durch das gezielte Arbeiten an den Faszien kann eine weitere Trainingsoptimierung betrieben werden mit dem Ziel, den Athleten für Höchstleistungen vorzubereiten, sowie zur Verletzungsprävention. Hierbei sind sowohl eine fasziengerechte Vorgehensweise im Rahmen der disziplinindividuellen Trainingsplanung empfehlenswert als auch das Training der
Faszien, also die Pflege der Faszien, an sich. Im Gegensatz zum Muskeltraining, bei dem anfangs schnelle Zuwächse zu verbuchen sind, verändern sich Faszien langsamer und kontinuierlicher. Erfolge im Faszientraining sind daher regelmäßig nur langfristig erzielbar. Es genügt allerdings, mit wesentlich geringeren Belastungsintervallen zu arbeiten. Zudem zahlt sich geduldige Regelmäßigkeit aus. Elastizität und Spannkraft im Fasziennetzwerk werden damit nachhaltig angelegt.

Eine Möglichkeit zur geräteunterstützten Faszienpflege ist z.B. das Training mit der „BlackRoll ${ }^{\circledR \lll . ~ D a b e i ~ w e r d e n ~ K o ̈ r p e r-~}$ stellen mittels einer Hartschaumrolle gezielt mobilisiert, indem mit dem Druck des eigenen Körpergewichts auf der Rolle gearbeitet wird. Dadurch können Verklebungen gelöst, Flüssigkeitsverschiebungen erwirkt und die Elastizität der faszialen Struktur positiv beeinflusst werden. Aktuelle Studien zur Wirkungsweise des Faszientrainings laufen derzeit im Institut für angewandte Trainingswissenschaften (IAT) in Leipzig.

Im Ergebnis ist festzustellen, dass sich die Erforschung des Faszienkomplexes inkl. aller detaillierten Wirkungsweisen zwar einerseits noch in den Anfängen befindet, andererseits jedoch praktische Erfahrungen und Therapieerfolge auf diesem Gebiet deutliche positive Ergebnisse aufzeigen und bereits so vielfach die positive Effektivität belegen.

Aus dem Blickwinkel des erfahrenen Osteopathen ist das FDM in der beschrieben Weise eine wirkungsvolle Ergänzung der klassischen Behandlungstechniken in der Osteopathie.

\section{Literatur}

1 Schwind P. Faszien- und Membrantechnik. 2. Aufl. München: Urban \& Fischer/Elsevier; 2009

2 Myers TW. Anatomy Trains. Myofasziale Leitbahnen. 2. Aufl. München: Urban \& Fischer/ Elsevier; 2010

3 Typaldos S. Orthopathische Medizin. 3. Aufl. Kötzting: Wühr; 2010

4 Römer F. Praktisches Lehrbuch zum Fasziendistorsionsmodell. 2. Aufl. Wolfenbüttel: Römer; 2014

5 Langer W, Hebgen E. Lehrbuch Osteopathie. Stuttgart: Haug; 2013

6 Still AT. The Philosophy and Mechanical Principles of Osteopathy. Kansas City, Missouri: Hudson-Kimberly; 1902

\section{Online}

http://dx.doi.org/10.1055/s-0034-1383004

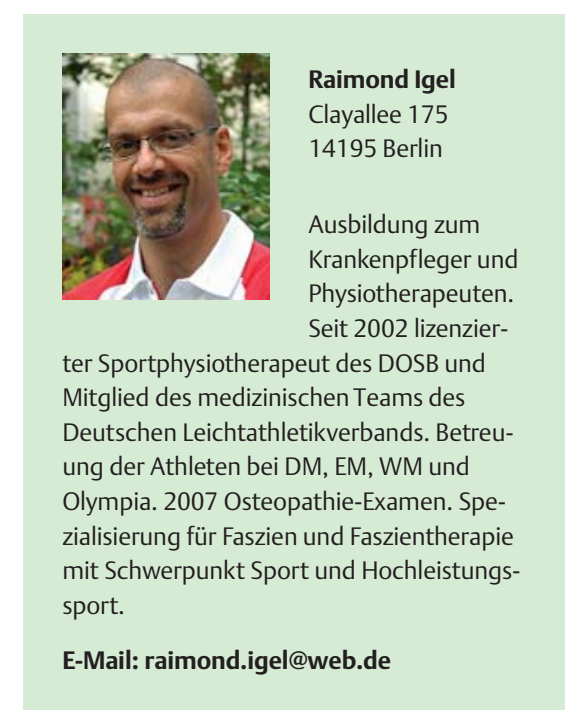

